\title{
Physician Ownership in der Privatpraxis: Pflegen und erhalten
}

Richard O. Binswanger

1 Binswanger RO. Ärztliche Führung. Schweiz Ärztezeitung. 2010;(91)21:835.

2 Hanson PS. Private Practice Trends in US Radiology, Imaging Management. 2009;9(1):16-21.

3 Lessmann W. RNR AG Persönliche Mitteilung.

4 www.fsb-spital.ch

5 www.vrnz.de

6 Wenger M. Schlossbergpraxis Frauenfeld. Persönliche Mitteilung.

Korrespondenz:

Dr. med. Richard O. Binswanger Führungsschule Bodensee Münsterlingen

Oberer Seeweg 9

CH-8597 Landschlacht

r.binswanger[at]bluewin.ch

www.fsb-spital.ch
In einem früheren Beitrag hat sich der Autor mit der Physician Leadership in Gesundheitsorganisationen auseinandergesetzt [1]. Jetzt geht es um Physician Ownership in der Privatpraxis. Aufgrund von Erfahrungen aus Kursen in Deutschland halte ich die Physician Ownership auch in der Schweiz langfristig für bedroht. Ich beziehe mich im Folgenden vor allem auf die Radiologie.

\section{Die Industrialisierung der Medizin}

Sie schreitet unablässig fort, ob wir sie wollen oder nicht. In den USA nahm der Anteil der Radiologiepraxen mit mehr als 30 Radiologen von 1990 bis 2007 von 5 auf 29 Prozent zu [2]. Die grösste Radiologiepraxis in Deutschland, die Radiologie Nordrhein AG (RNR AG), beschäftigt über 100 Radiologen [3]. Die Konsolidation der Privatradiologie ist in vollem Gang und nimmt rasch zu. Das bedeutet regionale und überregionale Zusammenschlüsse und Akquisitionen. Investmentgesellschaften versuchen, radiologische Unternehmen zu erwerben.

\section{Probleme grosser Partnerschaften}

In Deutschland sind die grossen Praxen als «Gesellschaft Bürgerlichen Rechts» (GBR) organisiert, was in der Schweiz der «Einfachen Gesellschaft» entspricht. Die Praxen kämpfen mit erheblichen Problemen: Alle Entscheidungen verlangen Einstimmigkeit der Teilhaber. Jeder Partner kann jeden Entscheid blockieren. Streit, Konflikt und Disput sind die Regel, nicht die Ausnahme. Governance, Strategie und Management

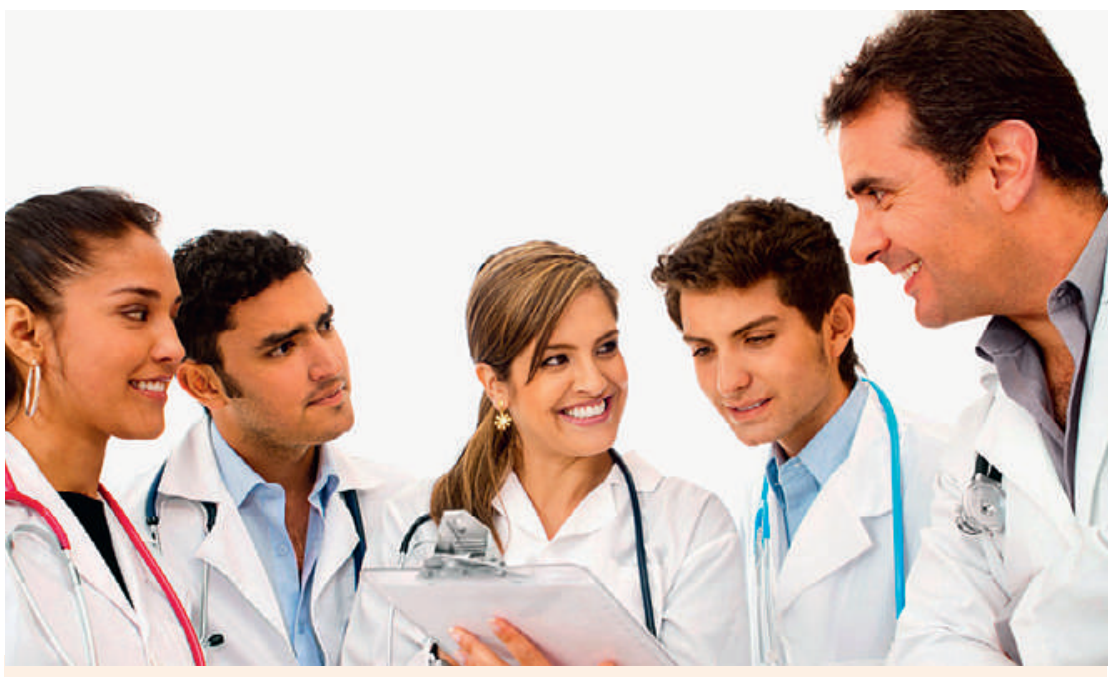

Ab einer bestimmten Grösse und Anzahl Ärzte sind Gruppenpraxen nicht mehr als einfache Gesellschaft führbar. leiden ernsthaft. Physician Leadership ist erratisch oder fehlt. Nachfolger sind schwierig zu finden. Physician Ownership wird zur Bürde.

\section{Auswege}

Unsere Führungsschule [4] leitet grosse Seminare für den Verbund Radiologischer und Nuklearmedizinischer Zentren VRNZ in Bayern [5]. Die Themen sind: Werte und Haltungen, Governance, Strategie, Leadership, Unternehmensstruktur, Rechtsform, Konsolidation, Zusammenschlüsse und Akquisitionen. Unter dem Einfluss der Kurse beginnen die Zentren sich zu professionalisieren, wandeln die GBRs in Kapitalgesellschaften um, zumeist als GmbH. Verhandlungen für Zusammenschlüsse sind im Gang. Governance und Leadership verbessern sich.

\section{Konsequenzen für die Ärzte}

Gruppenpraxen mit über 10 Teilhabern sind als einfache Gesellschaft nicht führbar [6]. Die Umwandlung in eine andere Gesellschaftsform, zumeist in eine Kapitalgesellschaft, ist notwendig. Jeder Partner besitzt jetzt nur noch eine kleine Menge von Anteilen. Sein Einfluss wird schwächer, aber Entscheidungen werden deblockiert und mit der Mehrheit der Stimmen gefällt. Das Unternehmensrisiko verteilt sich auf mehr Schultern. Das Management kann professionalisiert werden, die Führung muss aber bei den Ärzten bleiben.

\section{Aber}

Als die RNR AG aus mehreren Gesellschaften Bürgerlichen Rechts gegründet wurde, verkauften jüngere Teilhaber ihre Aktien an ältere [3]. Sie scheinen Geld $\mathrm{zu}$ benötigen, sind risikoscheu und bevorzugen ein fixes Einkommen. Das ist keine gute Entwicklung und dem Gedanken der Physician Ownership abträglich. Ärzte, die austreten, versuchen, ihre Anteile zu behalten. Denn sie sind eine sehr gute Geldanlage. Dagegen hilft nur ein fairer Gesellschaftsvertrag, der die Übertragung der Anteile an neueintretende Ärzte regelt unter Berücksichtigung angemessener Warteund Übergangsfristen.

\section{Fazit}

Physician Ownership in der Privatpraxis wird zunehmend schwierig zu bewerkstelligen. Schwierig zu erhalten. Schwierig, der nächsten Generation zu übergeben. Verkaufen Sie trotzdem Ihre Anteile nicht an Investoren oder andere. Physician Ownership verdient, erhalten zu bleiben. 